\title{
ALCUNE NOTE SULLA NOZIONE DI SPIEGAZIONE NELLE SCIENZE E IN FILOSOFIA
}

\author{
GIULIANO TORRENGO (*)
}

SunTo. - Quando spieghiamo qualcosa, usiamo ipotesi, ossia rappresentazioni, formulate in riferimento ad una teoria di sfondo. Ciò che rende tali rappresentazioni delle spiegazioni sono fatti nel mondo. Se, ad esempio, mi appello all'ipotesi che il secondo revisore è stato ingiusto per spiegare perché Claudio è arrabbiato, tale ipotesi è una (buona) spiegazione se e solo se il secondo revisore è stato ingiusto. Ne segue che anche se le teorie scientifiche possono sempre essere lette in senso strumentalista, nell'usarle per formulare ipotesi esplicative facciamo spesso appello, implicitamente o esplicitamente, a letture che vanno oltre il loro mero contenuto empirico.

$$
* * *
$$

ABSTRACT. - When we explain something we use hypotheses, that is representations, which we formulate on the basis of some background theory or other. What makes such representations explanations are facts in the world. If, for instance, I can appeal to the hypothesis that the second referee has been unfair as a way of accounting for why Claudio is upset. and such a hypothesis is a (good) explanation if and only if the second referee has been unfair. It follows that even if scientific theories can always be read in an instrumentalist sense, in using them to formulate explanatory hypotheses we often appeal, implicitly or explicitly, to readings of them that go beyond their empirical content.

\section{REALISMO E ANTIREALISMO}

Uno dei problemi filosofici tradizionali che sembra connettere in maniera profonda la riflessione metafisica e a priori alle scienze empiriche è il problema della differenza fra gli atteggiamenti realisti e antirealisti nei confronti di un certo ambito di discorso. Parlo di atteggiamenti o posizioni (teoriche), piuttosto che di teorie realiste o antirealiste, per-

(*) Centre for Philosophy of Time, Dipartimento di Filosofia "Piero Martinetti", Università di Milano, Italia. E-mail: giuliano.torrengo@unimi.it 
ché mentre una teoria metafisica può essere esplicitamente realista o antirealista rispetto a un certo ambito di discorso, all'interno delle scienze empiriche troviamo normalmente teorie che possiamo leggere sia in senso realista sia in senso antirealista. In altri termini, la distinzione realista/antirealista riguarda l'implicito di una teoria scientifica, il modo in cui viene compresa, piuttosto che il contenuto delle proposizioni di cui è costituita. L'idea qui è che esista sempre un contenuto empirico minimale delle teorie scientifiche, che è di per sé neutrale rispetto alle questioni metafisiche o più generalmente filosofiche, perché di per sé non ci impegna a credere che il mondo sia fatto in una certa maniera, al di là delle previsioni sulle osservazioni future che la teoria ci permette di fare. Possiamo dire che tale contenuto empirico sia il risultato di una lettura "strumentalista" della teoria, ossia quella lettura che la intende come una serie di enunciati che correlano istruzioni per manipolare sistemi a predizioni su osservazioni riguardanti gli stessi. E per questo motivo il dibattito sulla domanda su quale sia l'atteggiamento giusto (data una certa teoria $T$ e un ambito di discorso $D$ correlato ad essa) fra quello realista e quello antirealista è un dibattito che necessariamente esula contenuto empirico delle teorie, e in questo senso è - anche quando fisici, biologi e altri professionisti delle "scienze dure" ne sono coinvolti - eminentemente filosofico.

Di esterno al contenuto empirico di una teoria scientifica non c'è solamente l'atteggiamento o modo con cui viene compresa, ma anche il suo utilizzo. Le teorie ci forniscono apparati concettuali che possiamo usare per formulare ipotesi. Cruciale, per l'accumulo e il progresso scientifico stesso, è che queste ipotesi siano testabili, ossia esistano procedure sperimentali i cui esiti sappiamo interpretare in termini di conferma della teoria stessa. Ma altrettanto importante, almeno dal punto di vista filosofico, è che le teorie scientifiche si possano usare per formulare ipotesi esplicative - ossia ipotesi che possono essere viste come spiegazioni di certi fenomeni o fatti. Ora, l'utilizzo di una teoria scientifica per fare ipotesi testabili è una questione strettamente connessa al contenuto empirico della teoria, ed è quindi plausibile pensare che sia indipendente dall'atteggiamento realista o antirealista che si può avere nei confronti della teoria. La situazione è diversa però quando le ipotesi vengono formulate con lo scopo di spiegare, ossia quando si utilizza una teoria scientifica per fare ipotesi esplicative, come cercherò di argomentare in ciò che segue. 


\section{SPIEGAZIONE E RAPPRESENTAZIONE}

Proviamo a distinguere tipi di spiegazioni rispetto ai contesti in cui le troviamo e le teorie di sfondo che vengono usate nel formularle. Ci sono spiegazioni che possiamo chiamare ordinarie, come (A), che possiamo formulare semplicemente utilizzano la conoscenza di fatti comportamentali (se non mi sveglio rimarrò nel letto e non uscirò) e triviali (se non esco di casa in tempo, non posso arrivare in tempo a lavoro).

\section{(A)}

1) Perché Michele è arrivato in ritardo a lavoro?

2) Non ha sentito la sveglia

Ci sono spiegazioni che possiamo chiamare scientifiche, come (B), la cui formulazione richiede che teorie più sofisticate (nell'esempio, la chimica di base) vengano utilizzate.

\section{(B)}

1) Perché il sale in quel bicchiere non si scioglie più?

2) La soluzione è satura.

In fine, ci sono spiegazioni che possiamo chiamare filosofiche, come $(\mathrm{C})$, in cui usiamo una teoria metafisica, il perdurantismo nell'esempio, per formulare un'ipotesi esplicativa.

\section{(C)}

1) Come ho continuato ad esistere da ieri a oggi?

2) Distinte mie parti temporali si sono succedute durante l'intervallo di tempo in questione.

La lista non vuole essere esaustiva, ma solo esemplificativa di come in contesti diversi troviamo diverse spiegazioni all'opera, e di come diverse teorie di sfondo possano essere usate per formulare ipotesi che sono (o vogliono essere) spiegazioni. Nel linguaggio ordinario talvolta diciamo che un'ipotesi (o una teoria) spiega qualcosa, ma penso ci siano buoni motivi per pensare che tale modo di parlare sia in un certo senso impreciso, e che sia corretto identificare ipotesi esplicative e spiegazioni. 
Se mettiamo da parte le differenze fra casi come (A), (B) e (C) per quanto interessanti e profonde - e riflettiamo su quest'ultimo punto, possiamo vedere come la nozione di spiegazione sia connessa in modo stretto, ma non banale, a quella di rappresentazione. Se le spiegazioni sono ipotesi, allora sono un tipo di rappresentazione. Più precisamente, c'è una dipendenza esistenziale delle spiegazioni dalle rappresentazioni in generale: è difficile pensare ad un mondo in cui ci siano spiegazioni, ma non ci siano rappresentazioni, mentre sicuramente ci sono rappresentazioni che non sono spiegazioni, e potrebbe essere solo una contingenza che ci troviamo in un mondo dove alcune rappresentazioni sono spiegazioni. Se questi ragionamenti sono corretti, allora una spiegazione è un insieme di enunciati interpretati o altre entità di tipo rappresentazionale (come le proposizioni). Quali insiemi di enunciati interpretati contino come spiegazioni, e fra questi quali contino come buone spiegazioni è qualcosa su cui possiamo essere in disaccordo, anche se ci troviamo d'accordo sul fatto che una spiegazione è un insieme di enunciati interpretati.

\section{III.SPIEGAZIONE E IMPEGNO METAFISICO}

Anche se le spiegazioni sono rappresentazioni, ciò non vuol dire che ciò che "fa" di un enunciato una spiegazione, il meccanismo esplicativo sottostante, sia necessariamente qualcosa di rappresentazionale. In altri termini, anche se il "portatore" di una spiegazione è sempre un enunciato interpretato o più in generale un'entità rappresentazionale, quando ci chiediamo cosa rende un certo insieme di enunciati una (buona) spiegazione, la risposta a questa domanda non coinvolgerà necessariamente rappresentazioni o loro proprietà.

Questo è qualcosa che viene fuori anche nel modo in cui noi parliamo normalmente. Se io chiedo, ad esempio, perché Claudio è arrabbiato. E la risposta è che è arrabbiato perché il secondo revisore dell'articolo che aveva mandato alla rivista "peer reviewed" è stato particolarmente ingiusto nei suoi confronti, ciò a cui facciamo appello per dare una spiegazione (in questo caso una spiegazione "ordinaria", né scientifica né filosofica) sono fatti nel mondo. Che cos'è che spiega l'arrabbiatura di Claudio? È il fatto che il revisore abbia scritto certe cose in un certo contesto e così via. Anche se la spiegazione in questione è una 
rappresentazione (l'enunciato che rappresenta, appunto, il motivo dell'arrabbiatura di Claudio), ciò che la rende una spiegazione sono fatti che riguardano una persona (il revisore), il prodotto delle sue azioni e l'accesso che Claudio ne ha.

Spesso, dunque, quando diamo una spiegazione, anche se usiamo sempre rappresentazioni (le ipotesi che possiamo estrarre da una teoria di sfondo), ciò che spiega non sono le rappresentazioni o le loro proprietà intrinseche, ma sono fatti nel mondo, elementi non rappresentazionali. (C'è un senso di "spiegare" stando a cui siamo noi che spieghiamo; ma tale senso è sinonimo di "dare una spiegazione", e anche se siamo sempre noi a dare spiegazioni, sono i fatti nel mondo a rendere delle spiegazioni gli enunciati che usiamo nel dare spiegazioni, e in tal senso a spiegare.)

Se dunque affinché alcune ipotesi siano spiegazioni il mondo deve essere fatto in una certa maniera, sembra naturale pensare che accettare tale ipotesi come spiegazioni significhi ugualmente accettare che il mondo sia fatto in tale maniera. In altri termini, mentre accettare il solo contenuto empirico di una teoria non ci impegna ad accettare tesi metafisiche su come sia fatto il mondo al di là della sfera dell'osservabile, accettare una teoria come fonte di ipotesi esplicative ci impegna a tesi sul mondo che possono andare oltre il mero contenuto empirico della teoria.

\section{SPIEGAZIONE E CONTESTO}

Il fatto che accettare un'ipotesi come spiegazione porti con sé un impegno che va oltre al mero contenuto empirico dell'ipotesi non vuol dire che le spiegazioni non abbiano di per sé aspetti contestuali o sensibili ai nostri scopi e interessi. Quando io chiedo perché Claudio sia arrabbiato, il tipo di spiegazione richiesta dipende dal contesto. In un contesto ordinario, quello che chiedo è quali sono i fatti comportamentali rilevanti, grossomodo quelli che posso formulare data una qualche teoria di psicologia naïve di sfondo. $\mathrm{Ma}$ in un contesto diverso, ad esempio se sono interessato alle spiegazioni che possiamo dare sulla base delle nostre conoscenze neuro-scientifiche, la risposta non farebbe appello a fatti comportamentali, ma piuttosto a fatti che riguardano $\mathrm{i}$ neuroni di Claudio. 
In generale, il fatto che le spiegazioni richiedano che il mondo sia fatto in una certa maniera non vuol dire che essere una (buona) spiegazione non dipenda dai fini di chi fornisce una spiegazione e da quale teoria di sfondo sia rilevante per perseguire tali fini. Ciò vuol dire che se l'impegno che porta con sé l'accettazione di una spiegazione è sempre "metafisico" nel senso di andare oltre al mero contenuto empirico della teoria, non è detto che sia un impegno particolarmente interessante per i dibattiti filosofici che ricadono sotto l'etichetta di "metafisica" (o "metafisica della scienza"). Nel contempo, non è detto che un'ipotesi porti un impegno metafisico in questo senso più ristretto (come interessante per i dibattiti attuali) solo se la leggiamo come un'ipotesi come esplicativa di un qualche problema di un dibattito che ci interessa. In particolare, quando ci chiediamo quale atteggiamento (realista o antirealista) accettare una certa spiegazione porti con sé nei confronti di un certo ambito di discorso, facciamo qualcosa di più complicato. Finirò con qualche riflessione su questo punto.

\section{Spiegazione E MEtafisica}

Nel momento in cui discutiamo di teorie scientifiche e ci chiediamo se un certo atteggiamento sia realista o meno, facciamo una specie di doppio gioco. Da un lato, il contesto esplicativo è grossomodo quello della teoria scientifica a cui siamo interessati. Dall'altro, i criteri che consideriamo per valutare la spiegazione non sono (non solo, per lo meno) meramente empirici, nel senso che non riguardano solo la capacità di predire e l'adeguatezza con le osservazioni. Ciò che guardiamo sono piuttosto le "virtù teoretiche" che la teoria mostra quando la usiamo per formulare spiegazioni, se lette in una certa maniera: l'unità con altri ambiti del sapere, la semplicità, e il fatto che lette in tal modo le ipotesi forniscano maggiore intellegibilità ai meccanismi esplicativi sottostanti, ossia agli elementi che fanno la spiegazione (nel senso visto in precedenza).

Nella misura in cui le ipotesi esplicative non si limitano ai concetti e agli apparati teorici che sono strettamente legati al contenuto empirico di una teoria, ma richiedono teorie di sfondo più ampie, "leggere" una teoria scientifica in un certo modo significa leggerla insieme a qualche teoria metafisica, e quindi plausibilmente insieme a qualche impe- 
gno di tipo metafisico in senso stretto. Ad esempio, se dobbiamo spiegare come gli individui interagiscono fra di loro in un contesto quantistico, le nostre ipotesi diventano più esplicative nel momento in cui la risposta la diamo insieme anche a qualche punto più ampio e filosofico, ad esempio cosa sia un individuo e quali siano le condizioni di persistenza nel tempo degli individui. Possiamo rispondere a queste questioni metafisiche implicitamente o esplicitamente, ma in ogni caso è da questi aspetti non meramente empirici delle ipotesi he che arriva il potere esplicativo. Proprio perché le spiegazioni funzionano in una certa maniera, dunque, spesso l'appellarsi al fatto che un'ipotesi scientifica sia esplicativa, significa di fatto difendere una qualche tesi metafisica che abbiamo implicitamente o esplicitamente usato. 